\title{
ANÁLISE DA CAPACIDADE FUNCIONAL DOS IDOSOS DE PORTO ALEGRE E SUA ASSOCIAÇÃO COM AUTOPERCEPÇÃO DE SAÚDE
}

\author{
Maria Cristina Cardoso ${ }^{1}$ \\ Fernanda Martins Marquesan² \\ Zayanna Christina Lopes Lindôso ${ }^{3}$ \\ Rodolfo Schneider ${ }^{4}$ \\ Irenio Gomes 5 \\ Geraldo Atillio De Carliø
}

\footnotetext{
1 Fonoaudióloga, Doutora em Gerontologia Biomédica pela Pontifícia Universidade Católica do Rio Grande do Sul - PUCRS, Docente do Curso de Fonoaudiologia do Instituto Porto Alegre - IPA e da Universidade Federal de Ciências da Saúde de Porto Alegre. E-mail: mccardoso@via-rs.net.

2 Graduada em Educação Física, Mestre em em Ciências do Movimento Humano pela Universidade Federal do Rio Grande do Sul - UFRGS, Coordenadora do Curso de Especialização em Ciências da Saúde e do Esporte. E-mail: fernanda.marquesan@pucrs.br.

3 Terapeuta Ocupacional, Mestre em Gerontologia Biomédica pela Pontifícia Universidade Católica do Rio Grande do Sul - PUCRS, Docente do Curso de Graduação em Terapia Ocupacional da Universidade Federal de Pelotas - UFPEL. E-mail: zayanna@gmail.com.

4 Médico Geriatra, Doutor em Clínica Médica, Professor Adjunto do Programa de Pós-graduação em Gerontologia Biomédica da Pontifícia Universidade Católica do Rio Grande do Sul - PUCRS, Coordenador do Comitê de Ética em Pesquisa da Pontifícia Universidade Católica do Rio Grande do Sul-PUCRS. E-mail: rodolfohsch@terra.com.br.

5 Médico Neurologista, Doutor em Medicina, Professor Adjunto do Programa de Pós-graduação em Gerontologia Biomédica da Pontifícia Universidade Católica do Rio Grande do Sul - PUCRS, Coordenador do Departamento Científico de Neuroepidemiologia da Academia Brasileira de Neurologia. E-mail: ireniogomes@uol.com.br.

6 Farmacêutico Químico, Doutor em Farmácia e Bioquímica, Professor do Programa de Pós-graduação em Gerontologia Biomédica da Pontifícia Universidade Católica do Rio Grande do Sul - PUCRS, Coordenador do Laboratório de Bioquímica, Genética Molecular e Parasitologia do Instituto de Geriatria e Gerontologia da Pontifícia Universidade Católica do Rio Grande do Sul - PUCRS. E-mail: adecarli@pucrs.br.
} 
Introdução: A complexidade que abrange o processo do enveIhecimento e a saúde do idoso envolve o aumento da prevalência de doenças crônicas degenerativas em idades avançadas. Tais doenças acometem o idoso e interferem na sua capacidade funcional.

Objetivo: Este estudo tem como objetivo analisar a relação entre a necessidade de auxílio para a realização de atividades de vida diárias entre os idosos de Porto Alegre e a sua autopercepção de saúde frente às doenças crônicas degenerativas.

Metodologia: Trata-se de um estudo retrospectivo, com base em dados do estudo Multidimensional do Idoso de Porte Alegre, de 2005, realizado pelo Instituto de Geriatria e Gerontologia (IGG) da Pontifícia Universidade Católica do Rio Grande do Sul (PUCRS). Os dados foram analisados e as suas frequências foram comparadas entre a variável dependente necessidade de auxilio e as variáveis independentes: sexo, idade, autopercepção de saúde e doenças crônicas degenerativas, através do teste Qui-quadrado.

Resultados: Os resultados mostraram diferença significativa para a necessidade de auxílio na realização das atividades diárias em relação ao sexo com p=0,002. Quando comparada a faixa etária e as demais variáveis foi observado resultado estatisticamente significativo para atividades de casa $(p<0,001)$, ingestão de medicamentos $(p=0,010)$, higiene pessoal $(p<0,001)$ e para movimentarse $(p=0,004)$. Observou-se também que conforme o avanço da idade a necessidade de auxílio aumentou, sendo que após os 80 anos tal necessidade tornou-se mais importante. No que se refere à autopercepção de saúde, foram observados resultados significativos em relação às atividades de casa, tomar remédio, higiene e movimentar-se com p<0,001, exceto para alimentação. Quando comparados o diabetes, doenças cardíacas e isquemias cerebrais com as variáveis do estudo, os dados foram significativos ( $p<0,001)$ para quase todas as variáveis de necessidade de auxilio com exceção da higiene e alimentação em relação à diabetes, atividades de casa em relação à isquemia cerebral e higiene e alimentação em relação aos problemas cardíacos. Com relação à autopercepção de saúde, os dados foram significativos ( $p<0,001)$ para a necessidade de auxílio para atividades de casa, tomar remédio, higiene pessoal e movimentar-se.

Conclusão: Tais dados demonstram a importância da capacidade funcional do idoso como preditor do seu estado de saúde, pois 


\section{Introdução}

O processo de envelhecimento populacional no Brasil cresce de forma rápida devido à diminuição da taxa de fecundidade combinada com a queda da mortalidade (ALVES; RODRIGUES, 2005; NASRI, 2008). Num período de 40 anos, o Brasil teve o seu perfil demográfico modificado com relação à taxa de mortalidade, o mesmo observado em países em que a maioria da população é de jovens, cuja característica é de maioria de idosos. Frente a essa mudança de perfil, cita-se o aumento da prevalência de doenças mais complexas e consequentemente mais onerosas em termos de assistência, considerando que a clientela atingida por essas doenças pertencem a uma faixa etária mais avançada. Uma das justificativas para tal ônus seria a complexidade que envolve o processo de envelhecimento e a saúde do idoso que, por sua vez, pode apresentar uma multiplicidade de patologias, favorecendo o maior tempo de internação desses pacientes quando se compara aos pacientes jovens (IBGE, 2009).

Durante o envelhecimento, várias doenças podem acometer o idoso, de forma a interferir na sua saúde e capacidade funcional. Tais doenças, crônicas ou degenerativas, exigem uma ação multiprofissional em conjunto, que vise às necessidades de cada idoso, de forma a propiciar a manutenção e o aperfeiçoamento de sua capacidade funcional (LEVY, 2001; REICHEL; GALLO, 2001; PAPALÉO NETTO; KEIN; BRITO, 2006). A partir de uma abordagem diagnóstica e terapêutica multidimensional, ou seja, dispensando uma atenção abrangente à saúde do idoso de forma interdisciplinar e planejada, as doenças crônicas são controladas e as agudas tratáveis, preservando-se assim a autonomia, independência e promovendo uma melhor qualidade de vida ao idoso (PAPALÉO NETTO; KEIN; BRITO, 2006).

A avaliação da capacidade funcional dos idosos, portanto, tem se tornado cada vez mais importante, tanto no aspecto preventivo, quanto na 
assistência em níveis secundários, nos quais já se percebe a necessidade de tratamento do indivíduo. A capacidade de manter as habilidades físicas e mentais necessárias para uma vida independente e autônoma é um dos conceitos dados à capacidade funcional. Do ponto de vista da Saúde Pública, define-se a capacidade funcional como um novo conceito de saúde, mais adequado para instrumentalizar e operacionalizar a atenção à saúde do idoso (GORDILHO et al., 2000).

A incapacidade funcional do idoso pode interferir na sua autonomia (o indivíduo capaz de executar suas atividades por si próprio) e na sua independência - quando da realização das Atividades de Vida Diária (AVD'S) e Instrumentais (AIVD'S), além de influenciar, também, a sua qualidade de vida (FIRED; GURALNIK, 1997; BONARDI; AZEVEDO E SOUZA; MORAES, 2007). Alguns fatores relacionados à saúde dos idosos podem ser citados como importantes causas de limitações na realização de atividades diárias, dentre elas: acidente vascular encefálico (AVE), doenças articulares, insuficiência cardíaca (IC), doença pulmonar obstrutiva crônica (DPOC), diabetes mellitus (DM) e demências (CHAIMOWICZ, 1997; FIRED; GURALNIK, 1997; GIACOMIN et al., 2005; KAMPER, 2005).

A autopercepção de saúde é uma modalidade de pesquisa qualitativa realizada através da escuta de relatos, por meio de observações (in locum ou a posteriori), questionários ou entrevistas, constituída pela relação que o sujeito estabelece com os demais e pela forma como é percebido pelos outros. As observações são utilizadas em historiografias, podendo incluir a análise de documentos. Nos questionários são considerados, somente, os relatos narrados pelos sujeitos, cujo objetivo é apreender o que cada sujeito ou grupo atribui aos eventos de sua vida. O procedimento básico da coleta de dados se dá por uma entrevista aberta, na qual se pede ao sujeito que relate sobre algum aspecto de sua vida. A entrevista se inicia com uma "pergunta chave", que "no decorrer da conversa o entrevistador é livre para fazer perguntas no sentido de clarificar ou aprofundar pontos mais diretamente ligados ao objeto de estudo" (GLAT; PLETSCH, 2009).

A autopercepção de saúde utilizada como uma avaliação subjetiva das condições de saúde em resposta à questão: "Como o senhor (a) avalia a sua saúde?", é amplamente reconhecida como um indicador global de saúde e utilizada em vários estudos de diferentes países como um preditor de mortalidade, cujas pesquisas epidemiológicas vêm ganhando um importante espaço (BENYAMINI et al., 2003; DEEG; BATH, 2003; DEEG; KRIEGSMAN, 2003; SPIERS et al., 2003; SILVA; MENEZES, 2007). Segundo Deeg e Barth (2003), a maneira de responder a esta pergunta está correlacionada com o 
comportamento de saúde e, assim, a autopercepção de saúde apresenta-se como uma variável complexa que capta as múltiplas dimensões da relação entre a saúde física e outras características de cunho pessoal e social. De acordo com Damián, Pastor-Barruso e Valderroma-Gama (2008), tal questionamento é muito consistente na sua capacidade de predizer a mortalidade, a perda funcional e também tem sido associado com o envelhecimento bem sucedido, pois um envelhecimento saudável depende da interação multidimensional de vários fatores, como afirmam Alves e Rodrigues (2005).

A partir de tal questionamento, este estudo tem como objetivo analisar a relação entre a necessidade de auxílio para as atividades de vida diária de idosos do município de Porto Alegre com a autopercepção de saúde e doenças crônicas degenerativas.

\section{Metodologia}

Esta pesquisa foi desenvolvida com base em dados do Estudo Multidimensional dos Idosos de Porto Alegre (EMIPOA), realizado pelo Instituto de Geriatria e Gerontologia (IGG) da Pontifícia Universidade Católica do Rio Grande do Sul (PUCRS), no período de dezembro de 2005 a novembro de 2006. A amostragem foi aleatória e estratificada por região censitária, sendo a amostra constituída por 1078 idosos residentes do município de Porto Alegre, com idade igual ou superior a 60 anos e que concordaram em participar da pesquisa (EMIPOA). O critério de exclusão foi a impossibilidade de responder ao questionário.

Os idosos foram entrevistados em sua residência por um profissional treinado do Serviço Social da PUCRS, utilizando um questionário com dados demográficos, sócio-econômicos e de saúde. Esse questionário foi elaborado por pesquisadores participantes do EMIPOA com base no estudo sobre o Perfil dos Idosos do Rio Grande do Sul realizado em 1995 (que validou o questionário na ocasião), na qual diversas universidades e setores públicos participaram da pesquisa, inclusive a PUCRS. No presente estudo foram analisadas as questões referentes ao sexo, faixa etária, autopercepção de saúde, presença de doenças crônicas degenerativas (pressão alta, diabetes, isquemia cerebral, problemas do coração) e necessidades de auxílio para as atividades de vida diária.

Os dados foram digitados em ACESS 2000 e analisados utilizando o pacote estatístico SPSS versão 17. As comparações de frequências de necessidades de auxílio com as diversas variáveis estudadas foram realizadas 
através do teste do Qui-quadrado de Pearson. Foram consideradas associações significativas aquelas com valores de $\mathrm{p} \leq 0,05$.

\section{Resultados}

Foram estudados 1078 idosos, sendo que desses, 71,8\% do sexo feminino e $28,2 \%$ do sexo masculino, com idade entre 60 e 95 (idade média de 71,8 anos e dp $\pm 7,8)$. Apenas 9,5\% dos idosos referiram saúde má ou péssima, $51,8 \%$ pressão alta, $17,6 \%$ diabetes, $4,8 \%$ isquemia cerebral e $27,2 \%$ problemas de coração. Em relação à necessidade de auxílio, 26,1\% referiram precisar de ajuda para as atividades da casa, 6,2\% para tomar remédios, 4,1\% para higiene, 2,1\% para alimentação e 5,2\% para movimentação.

A Tabela 1 mostra as frequências de necessidades de auxílio de acordo com sexo, faixa etária, autopercepção de saúde e presença de doenças crônicas. Em relação ao sexo, foi evidenciada uma diferença significativa apenas para a necessidade de auxílio na realização de atividades de casa $(\mathrm{p}=0,002)$, sendo observada maior necessidade entre as mulheres $(28,7 \%)$. Nos homens esse percentual foi de $19,3 \%$.

Tabela 1 - População estudada e freqüência de idosos que referiram necessidade de auxilio para atividades de casa, tomar remédio, higiene, alimentação e movimentação, de acordo com os dados demográficos, a autopercepção de saúde e a presença de doenças crônicas degenerativas, em 1078 idosos residentes em Porto Alegre.

\begin{tabular}{|c|c|c|c|c|c|c|}
\hline \multirow[b]{2}{*}{ Variável } & \multirow{2}{*}{$\begin{array}{c}\text { População } \\
\text { N (\%) }\end{array}$} & \multicolumn{5}{|c|}{ Necessidade de auxilio para } \\
\hline & & $\begin{array}{l}\text { Atividades } \\
\text { de casa \% }\end{array}$ & $\begin{array}{c}\text { Tomar } \\
\text { remédio \% }\end{array}$ & $\begin{array}{l}\text { Higiene } \\
\%\end{array}$ & $\begin{array}{c}\text { Alimentação } \\
\%\end{array}$ & $\begin{array}{c}\text { Movimentação } \\
\%\end{array}$ \\
\hline \multicolumn{7}{|l|}{ Sexo } \\
\hline Masc & $304(28,2)$ & 19,3 & 5,7 & 4,6 & 1,7 & 5,6 \\
\hline Fem & $774(71,8)$ & 28,7 & 6,4 & 3,9 & 2,3 & 5,1 \\
\hline$P$ & & 0,002 & 0,663 & 0,593 & 0,481 & 0,724 \\
\hline \multicolumn{7}{|c|}{ Faixa Etária } \\
\hline $60-69$ & $458(42,5)$ & 19,8 & 4,8 & 2,2 & 2,0 & 3,7 \\
\hline $70-79$ & $420(39)$ & 27,0 & 5,5 & 2,9 & 1,4 & 4,8 \\
\hline $80 \mathrm{ou}+$ & $200(18,6)$ & 38,4 & 10,7 & 11,1 & 4,0 & 9,5 \\
\hline$P$ & & $<0,001$ & 0,010 & $<0,001$ & 0,196 & 0,004 \\
\hline
\end{tabular}


(...continuação)

Autopercepção de saúde

\begin{tabular}{|c|c|c|c|c|c|c|}
\hline Ótima/boa & $510(47,3)$ & 20,0 & 3,2 & 2,0 & 1,0 & 2,0 \\
\hline Regular & $466(43,2)$ & 28,5 & 7,1 & 5,0 & 3,2 & 6,5 \\
\hline Má/péssima & $102(9,5)$ & 45,5 & 17,2 & 11,0 & 3,0 & 16,0 \\
\hline$P$ & & $<0,001$ & $<0,001$ & $<0,001$ & 0,044 & $<0,001$ \\
\hline \multicolumn{7}{|l|}{ Pressão Alta } \\
\hline Não & $506(46,9)$ & 23,9 & 4,8 & 4,6 & 2,0 & 4,4 \\
\hline Sim & $558(51,8)$ & 28,2 & 7,4 & 3,6 & 2,4 & 6,0 \\
\hline$P$ & & 0,111 & 0,074 & 0,440 & 0,663 & 0,236 \\
\hline \multicolumn{7}{|l|}{ Diabetes } \\
\hline Não & $865(80,2)$ & 24,2 & 4,3 & 3,6 & 1,6 & 4,1 \\
\hline Sim & $190(17,6)$ & 36,2 & 14,4 & 6,4 & 4,3 & 10,7 \\
\hline$P$ & & 0,001 & $<0,001$ & 0,081 & 0,023 & $<0,001$ \\
\hline \multicolumn{7}{|c|}{ Isquemia Cerebral } \\
\hline Não & $\begin{array}{r}1023 \\
(94,9) \\
\end{array}$ & 25,3 & 5,6 & 3,4 & 1,7 & 4,3 \\
\hline Sim & $52(4,8)$ & 41,2 & 17,3 & 15,4 & 9,6 & 21,2 \\
\hline$P$ & & 0,012 & 0,001 & $<0,001$ & $<0,001$ & $<0,001$ \\
\hline \multicolumn{7}{|c|}{ Problema de Coração } \\
\hline Não & $754(69,9)$ & 24,0 & 4,5 & 3,3 & 1,7 & 3,6 \\
\hline $\operatorname{Sim}$ & $293(27,2)$ & 32,5 & 10,7 & 6,2 & 3,1 & 9,7 \\
\hline$P$ & & 0,005 & $<0,001$ & 0,036 & 0,167 & $<0,001$ \\
\hline TOTAL & $1078(100)$ & 26,1 & 6,2 & 4,1 & 2,1 & 5,2 \\
\hline
\end{tabular}

Resultados a partir do Teste Qui-quadrado com intervalo de confiança de 95\%

No que se refere à idade, foi observada maior frequência de necessidades de auxílio conforme aumento da faixa etária, sendo o aumento mais significativo após os 80 anos. Resultados estatisticamente significativos foram observados entre a faixa etária e atividades de casa, tomar remédio, higiene e alimentação, exceto para alimentação.

A frequência de necessidade de auxílio para as atividades de casa foi de $20 \%$ para os idosos que referiram saúde ótima ou boa e de $45 \%$ para aqueles que referiram saúde má ou péssima. Essa diferença também é observada para 
tomar remédio ( $3 \%$ vs. $17 \%$ ), higiene ( $2 \%$ vs. $11 \%$ ), alimentação (1\% vs. $3 \%$ ) e movimentação ( $2 \%$ vs. $16 \%)$, todas com $p<0,05$.

Ao estudar as doenças crônicas, observou-se que a presença de pressão alta não se associou com necessidades de auxílio para as atividades habituais. A presença de diabetes e isquemia cerebral (IC) se associou com maior frequência a todas as necessidades de auxílio estudadas $(\mathrm{p}<0,05)$. Já frente aos problemas cardíacos, os dados foram estatisticamente significativos para as atividades de casa $(\mathrm{p}=0,005)$, para ingerir medicamentos $(\mathrm{p}<0,001)$, para higiene $(\mathrm{p}=0,036)$ e para movimentar-se $(\mathrm{p}<0,001)$ e não para alimentação $(\mathrm{p}=0,167)$.

\section{Discussão e Conclusão}

A Sociedade Americana de Geriatria define o termo "fragilidade" como sendo o declínio de reservas e resistência aos agentes estressores que agem na homeostase do organismo e acarretam em declínio de múltiplos sistemas fisiológicos (FRIED et al., 2001; BONARDI; AZEVEDO E SOUZA; MORAES, 2007). Conforme a idade avança, pode-se observar um declínio na capacidade funcional do indivíduo. Os idosos especificamente, podem tornar-se portadores de fragilidades que influenciam na sua funcionalidade e consequentemente afetam seu desempenho em suas AVD'S e AIVD'S.

No que se refere à realização das AVD'S e AIVD'S, alguns estudos já evidenciaram, em seus resultados, que quanto maior a idade, maior pode ser o nível de dependência do idoso na execução dessas atividades. Como exemplo, tem-se o estudo de Alves et al. (2008), realizado no município de Juiz de Fora (MG), no qual foram entrevistados 655 idosos, sorteados aleatoriamente na base do SIAB (Sistema de Informação da Atenção Básica), com o objetivo de conhecer suas características e avaliar sua capacidade funcional. Os resultados desse estudo apontaram que $58,2 \%$ dos idosos apresentaram algum grau de dependência e esse percentual se elevou com o avanço da idade. Esses achados permitiram aos autores observar que as atividades contidas nas AVD'S e AIVD'S tornam-se muito importantes na manutenção da capacidade funcional.

As características do referido estudo não se diferem dos resultados aqui encontrados e reforçam a importância para o idoso em se manter ativo e com máxima independência possível na realização de suas atividades diárias. Isso poderia afastar ou amenizar o surgimento ou agravo de possíveis comorbidades que o idoso possa adquirir. 
Além do processo normal de envelhecimento, as doenças crônicas e agudas podem desencadear grandes comorbidades e altas taxas de mortalidade no idoso. As comorbidades variam de acordo com o estado funcional do idoso antes do evento clínico, assim como pela localização e extensão da afecção que o acomete. Busby-Whitehead (2001) afirma que tal heterogeneidade entre os indivíduos idosos varia o estado funcional dos mesmos e que o desenvolvimento de processos patológicos pode afetar a capacidade cardíaca e a atividade física adversamente, desencadeando um comportamento sedentário e contribuindo ainda mais para o declínio do estado funcional relacionado à idade.

A baixa capacidade cardiorrespiratória e a pouca atividade física são fatores de risco independentes para o acometimento de doenças arteriais coronarianas assim como a atividade física evidencia uma relação inversa frente à pressão arterial e frente à DM (BUSBY-WHITEHEAD, 2001).

Os resultados demonstram um estado funcional diminuído dos idosos de Porto Alegre acometidos por doenças crônicas degenerativas frente à realização de atividades diárias. Fato que restringe a sua autonomia e independência e acarreta numa qualidade de vida má ou péssima, confirmada por sua autopercepção de saúde.

A necessidade de auxílio na realização de atividades diárias foi considerada quanto ao grau de dependência do idoso para a alimentação, podendo significar a necessidade de ajuda de outra pessoa ou a impossibilidade em realizar a tarefa. Essa necessidade, ou impossibilidade, foi descrita nos parâmetros de mensuração da incapacidade funcional revisada por Alves, Leite e Machado (2008).

A percepção de necessidade de auxílio nas atividades diárias consideradas estatisticamente significativas, como comorbidades relacionadas à $\mathrm{DM}$, à IC e aos problemas de coração, gera uma incapacidade funcional e nos remete a possibilidade de dificuldade motora desencadeada pela doença cérebro vascular e evidenciada pela paresia (com graus diferenciados de fraqueza muscular), déficits cognitivos, de função executiva e do comprometimento cardiorrespiratório, como analisado por Andre (2006) e Rizzetti e Trevisan (2008). A associação à DM pode ser explicada pelos múltiplos fatores relacionados principalmente às complicações vasculares e neuropáticas, como concluíram Alves et al. (2007).

A necessidade e a percepção de auxílios mostrados nos estudos anteriormente citados reforçam a importância da interdisciplinaridade no atendimento ao idoso. Terapeutas ocupacionais, fonoaudiólogos e educadores 
físicos são exemplos de profissionais que atuam junto à essa clientela de forma ampla.

O terapeuta ocupacional irá focar-se no desempenho do idoso em suas atividades e tarefas diárias através do uso terapêutico dessas e o treino de AVD'S e AIVD'S para manter o idoso o mais independente possível considerando seus aspectos motores, cognitivos, mentais e sensoriais. $\mathrm{O}$ fonoaudiólogo busca identificar as dificuldades geradas pela limitação frente a sua autoalimentação, a ingestão de medicamentos e a compreensão e o uso da linguagem necessárias no seu dia a dia, reabilitando as funções orais e de linguagem, propiciando a melhoria da funcionalidade do idoso. Esse profissional também fornece subsídios ao cuidador quanto às funções orais e posturas ideais para alimentação, dessa forma, qualifica os serviços prestados no auxílio ao idoso, ou mesmo, previne a instalação de um distúrbio, como o encontrado em Dias e Cardoso (2009). Já o educador físico, pode estimular o idoso na prática de exercícios físicos regulares e direcionados ao seu estado de saúde geral. As medidas adotadas por esses profissionais permitem ao idoso manter-se ativo, excluindo-se comportamentos sedentários ou de descuidos com a saúde que podem influenciar na sua capacidade funcional, conforme descrito por Whitehead (2001) anteriormente.

O estudo caracteriza maior necessidade de auxílio do idoso de Porto Alegre para realizar as atividades de casa, seguidas pela movimentação e pelo auxílio para a higiene pessoal, quando associados às doenças de DM, IC e cardíacas. Observam-se maiores índices de incapacidade funcional e a geração de dependência frente à IC.

As dificuldades de ingerir medicamentos e alimentação aparecem como dependentes de auxílio em uma proporção maior frente à IC, seguida pelos idosos acometidos por DM e doenças cardíacas, com dados estatisticamente significativos (excetuando-se a alimentação frente às doenças cardíacas). De acordo com André (2006), as dificuldades de deglutição ocorrem em 25 a 30\% dos pacientes na fase aguda do AVE, dos quais $50 \%$ podem apresentar sinais de aspiração traqueal. A proporção de disfagia em idosos é de 70 a $90 \%$, decorrente do processo de envelhecimento e/ou por acometimento neurológico (SANTORO; BOHADANA; TSUJI, 2003; FIORESE; BILTON, 2004; ALVES et al., 2007).

No estudo realizado por Troncon et al. (2001), encontra-se uma prevalência maior de disfagia entre os diabéticos que nos controles, podendo indicar distúrbios da peristalse esofagiana, decorrente do comprometimento da inervação autonômica do esôfago. Os autores desse estudo ressaltaram a queixa de disfagia como comum na população de diabéticos. 
Os resultados encontrados com relação à necessidade de auxílio para as atividades de vida diária e a autopercepção de saúde são corroborados na literatura (BENYAMINI et al., 2003; DEEG; BATH, 2003; DEEG; KRIEGSMAN, 2003; SPIERS et al., 2003; DAMIÁN; PASTOR-BARRUSO; VALDERROMAGAMA, 2008) e apresentam um aspecto importante da relação que se estabelece entre o grau de independência para as atividades de vida diária e a percepção que o idoso tem do seu estado de saúde.

De forma geral, pode-se concluir que os resultados demonstram a importância que a capacidade funcional do idoso representa, visto como um preditor do seu estado de saúde, pois os resultados encontrados contemplam que, enquanto a necessidade de auxílio para as atividades de vida diária do idoso aumentam, piora a sua autopercepção de saúde e o seu estado real de saúde.

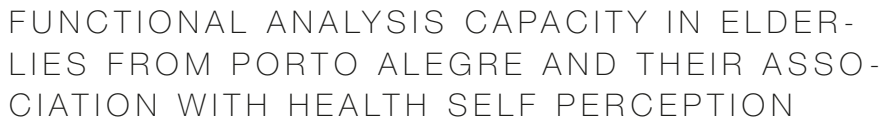

\section{abstract}

Introduction: The complexity that covers the process of ageing and elderly health involves increasing prevalence of chronic degenerative diseases in advanced ages. Such diseases that happen to the elderly interfere with their functional capacity.

Objective: The aim of this study is to examine the relationship between the need for aid for daily life activities between the elderly of Porto Alegre and their self perception of health to chronic degenerative diseases.

Methodology: It is a retrospective study based on data from the Porto Alegre Elderly Multidimensional study of 2005. The data were analyzed and their frequencies were compared among the perception of health variables, the need for aid and chronic degenerative diseases by the Chi square test.

Results: The results show significant difference to the need of assistance in carrying out daily activities for sex $(p=0.002)$, different age groups $(p<0.001)$, ingestion of medicines $(p=0.010)$, personal hygiene $(p<0.001)$ and for movements $(p=0.004)$. Chronic diseases, diabetes, heart disease and brain's ischemia were significant data for almost all variables of aid needs. About health self percep- 
tion were significant data $(\mathrm{p}<0.001))$ to the need for aid for activities, take medicine, personal hygiene and movements.

Conclusion: Such data demonstrate the importance of elderly functional capacity as a predictor of their state of health and the need for increasing aid, making their self perception health and their actual state worse.

Keywords

Elderly. Chronic Disease. Health Self perception. Healthcare Nee-

ds. Elderly Health.

\author{
referências
}

ALVES, Luciana Correia; LEIMANN, Beatriz Consuelo Quinet; VASCONCELOS, Maria Estrela Lopes; CARVALHO, Marilia Sá; VASCONCELOS, Ana Glória Godoi; FONSECA, Thaís Cristina Oliveira da; LEBRÃO, Maria Lúcia; LAURENTI, Ruy. A influencia das doenças crônicas na capacidade funcional dos idosos do Município de São Paulo, Brasil. Cademos de Saúde Pública, São Paulo, v. 23, n. 8, p. 1924-1930. ago. 2007.

ALVES, Luciana Correia; LEITE, luri da Costa; MACHADO, Carla Jorge. Conceituando e mesurando a incapacidade funcional da população idosa: uma revisão de literatura. Ciência e saúde coletiva, Rio de Janeiro, v. 23, n. 4, p. 1199-1207, jul./ago. 2008.

ALVES, Marcio Jose Martins; RIBEIRO, Luiz Claudio; MILANE, Juliana Lana; ALMEIDA, Andreia Munck de. Perfil da Capacidade Funcional do Idoso. Anais, XVI Encontro Nacional de Estudos Populacionais, Caxambu, 2008

ALVES, Luciana Correia; RODRIGUES, Roberto Nascimento. Determinantes da autopercepção de saúde entre idosos do Município de São Paulo, Brasil. Revista Panamericana de Salud Publica, Washington, v. 17, n. 5, supl. 6, p. 333-341. May, jun. 2005.

ANDRÉ, Charles. Manual do AVC. Rio de Janeiro: Livraria e Editora Revinter Ltda, 2006. $232 \mathrm{p}$.

BENYAMINI, Yael; BLUMSTEIN, Tzvia; LUSKY, Ayala; MODAN, Baruch. Gender Differences in the Self-Rated Health-Mortality Association: Is It Poor Self-Rated Health That Predicts Mortality or Excellent Self-Rated Health That Predicts Survival. The Gerontologist, Washington, v. 43, n. 3, p. 396-405, jun. 2003.

BONARDI, Gislaine; SOUZA, Valdemarina Bidone Azevedo; MORAES, João Feliz Duarte de. Incapacidade funcional e idosos: um desafio para os profissionais de saúde. Scientia Medica, Porto Alegre, v. 17, n. 3, p.138-144; 2007.

BUSBY-WHITEHEAD, Jan. Exercícios físicos para idosos. In: GALLO, Joseph J; BUSBY-WHITEHEAD, Jan; RABINS, Peter V; SILLIMAN, Rebecca A; MURPHY, John B. Reichel - assistência ao idoso, aspectos clínicos do envelhecimento. Rio de Janeiro: Editora Guanabara Koogan; 2001. p. 109-113.

CHAIMOWICZ, Flávio. A saúde dos idosos brasileiros às vésperas do século XXI: problemas, projeções e alternativas. Revista de Saúde Pública, São Paulo, v. 31, p. 184 200, abr. 1997 
DAMIÁN, Javier; PASTOR-BARRUSO, Roberto; VALDERROMA-GAMA, Emiliana. Factors associated with self-rated health in older people living in institutions. Biomedical Central Geriatrics, London, v. 8, p.1-6, feb. 2008.

DEEG, Dorly J. H.; BATH, Peter A. Self-rated health, gender and mortality in older person: Introduction to a special section. The Gerontologist, Washington, v. 43, n. 3, p. 369-371, jun. 2003.

DEEG, Dorly J. H.; KRIEGSMAN, Didi M.W. Concepts of Self-Rated Health: Specifying the Gender Difference in Mortality Risk. The Gerontologist, Washington, v. 43 n. 3, p. 376-386, jun. 2003.

DIAS, Berenice Klimuk Pereira; CARDOSO, Maria Cristina de Almeida Freitas. Características da função da deglutição em um grupo de idosas institucionalizadas. Estudos interdisciolinares sobre o envelhecimento, Porto Alegre v. 14, n. 1, p. 107-124, jun. 2009.

FIORESE, Ana Carolina; BILTON, Tereza Loffredo. Estudos das alterações de maior ocorrência nas fases oral e faríngeas da deglutição, entre 20 e 93 anos de idade, avaliados pela videofluroscopia. Distúrbios da Comunicação, São Paulo, v. 16, n. 3, p. 301-312, dez. 2004.

FRIED, Linda P; GURALNIK, Jack M. Disability in older adults: evidence regarding significance, etiology, and risk. Joumal of American Geriatrics Society Los Angeles, v. 45, p. $92-100.1997$.

FRIED, Linda P; TANGEN, Catherine M; WALSTON, Jeremy; NEWMAN, Anne B; HIRSCH, Calvin; GOTTDIENER, John; SEEMAN, Teresa; TRACY, Russell; KOP, Willem J; BURKE, Gregory; McBURNIE, Mary Ann. Frailty in older adults: evidence for a phenotype. The Joumals of Gerontology series A, Washington, v. 56, n. 3, p. 146-156, mar. 2001

GIACOMIN, Karla C; UCHÔA, Elizabeth; FIRMO, Josélia AO; LIMA-COSTA, Maria Fernanda. Projeto Bambuí: um estudo de base populacional da prevalência e dos fatores associados à necessidade de cuidador entre idosos. Cademos de. Saúde Pública, São Paulo, v. 21, n. 1, p. 80-91. jan./fev. 2005.

GLAT, Rosana; PLETSCH, Márcia Denise. O método de história de vida em pesquisas sobre auto-percepção de pessoas com necessidades educacionais especiais. Revista Educação Especial, Santa Maria, v. 22, n. 34, p. 139-154, maio/ago. 2009.

GORDILHO, Adriano; SÉRGIO, Joäo; SILVESTRE, Jorge; RAMOS, Luiz Roberto; FREIRE, Margarida Paes Alves; ESPINDOLA, Neidil; MAIA, Renato; VERAS, Renato; KARS$\mathrm{CH}$, Úrsula. Desafios a serem enfrentados no terceiro milênio pelo setor saúde na atenção integral ao idoso. Rio de Janeiro: Universidade Aberta da Terceira Idade - UnATl; 2000. p. 1-86.

Instituto Brasileiro de Geografia e Estatística - IBGE. IBGE divulga Indicadores Demográficos e de Saúde. [periódico na Internet] nov. 2011. Disponível em: <http://www.ibge.gov $\mathrm{br} /$ home/presidencia/noticias/noticia_visualiza.php?id_noticia=1445\&id_pagina=l> Acesso em: 10 nov. 2011.

KAMPER, Adriaan M; STOTT, David J; HYLAND, Michael; MURRAY, Heather M; FORD, lan. Predictors of functional decline in elderly people with vascular risk factors or disease. Age and Ageing, London, v. 34, n. 5, p. 450-455, out. 2005.

LEVY, Susan M. Avaliação multidimensional do paciente idoso. In: GALLO, Joseph J: BUSBY-WHITEHEAD, Jan; RABINS, Peter V; SILLIMAN, Rebecca A; MURPHY, John B. Reichel - assistência ao idoso, aspectos clínicos do envelhecimento. Rio de Janeiro: Editora Guanabara Koogan; 2001. p. 15-29.

NASRI, Fabio. O envelhecimento populacional no Brasil. Einstein: educação continuada em saúde, São Paulo, v. 6, supl. 1, p. S4-S6. 2008 
PAPALÉO NEITO, Matheus; KLEIN, Eva Lucila; BRITO, Francisco Carlos de. Avaliação geriátrica multidimensional. In: CARVALHO FILHO, Eurico Thomaz de; PAPALÉO NETTO, Matheus, Editores. Geriatria - fundamentos, clínica e terapêutica. São Paulo: Editora Atheneu; 2006. p. 73-85.

REICHEL, William; GALLO, Joseph J. Princípios fundamentais da assistência ao idoso. In: GALLO, Joseph J; BUSBY-WHITEHEAD, Jan; RABINS, Peter V; SILLIMAN, Rebecca A; MURPHY, John B. Reichel - assistência ao idoso, aspectos clínicos do envelhecimento. Rio de Janeiro: Editora Guanabara Koogan; 2001. p. 03-14.

RIZZEITI, Danize Aparecida; TREVISAN, Claudia Morais. Avaliação da capacidade funcional em pacientes portadores de seqüelas de AVC participantes do projeto hidrocinesioterapia aplicada às patologias neurológicas do idoso. Revista Saúde, Santa Maria, v. 34a, n. 1, supl, 2, p. 32-36, dez. 2008.

SANTORO, Patrícia Paula; BOHADANA, Saramira Cardoso; TSUJI, Domingos Hiroshi. Fisiologia da deglutição. In: CAMPOS, Carlos Alberto Herrerias de; COSTA, Henrique Olavo de Olival. Tratado de Otominolaringologia. São Paulo: Roca; 2003. v. 1, p. 768-782.

SILVA, Thiago Rodrigues da; MENEZES, Paulo Rossi. Autopercepção de saúde: um estudo com idosos de baixa renda de São Paulo / Self-rated health: a study in an elderly low income population of São Paulo. Revista de Medicina, São Paulo, v. 86, n. 1, p. 28-38. jan./mar. 2007.

SPIERS, Nicola; JAGGER, Carol; CLARKE, Michael; ARTHUR, Antony. Are Gender Differences in the Relationship Between Self-Rated Health and Mortality Enduring? Results From Three Birth Cohortsin Melton Mowbray, United Kingdom. The Gerontologist, Washington, v. 43, n. 3, p. 406-411, jun. 2003.

TRONCON, Luiz Ernesto de Almeida; LOPES, Rony Peterson; SIMÃO, Marcelo Novelino; ROSA-e-SILVA, Lucilene; NOBRE-e-SOUZA, Miguel Angelo; FOSS, Milton Cesar. Freqüência de sintomas digestivos em pacientes brasileiros com diabetes mellitus. Revista da Associação Médica Brasileira, São Paulo, v. 47, n. 2, p. 157-164, abr./jun. 2001.

Recebido: 06/12/2010

$1^{a}$ Revisão: 28/01/2011

2a Revisão: 23/02/2011

3a Revisão: 27/04/2011

Aceite Final: 24/05/2011 